Methods May through November 2010, we assessed serosorting practices and beliefs among MSM seeking STI testing at an urban LGBT health center. We compared serosorting practices and beliefs by HIV status and partner characteristics with Pearson $\chi^{2}$ tests. Sexual behaviours, partner characteristics, and HIV status were based on self-report.

Results 705 HIV-negative and 88 HIV-positive MSM completed the assessment. Median time since last HIV test was 7.5 months; nearly a third had not been tested in the last year. Overall, $53 \%$ reported no UAI, $27 \%$ reported seroconcordant UAI, and 18\% reported serodiscordant UAI with last sex partner. UAI was more common with seroconcordant partners than with serodiscordant partners (55\% vs $37 \%, p<0.001) ; 23 \%$ of HIV-positive and $17 \%$ of HIV-negative men reported serodiscordant UAI. $81 \%$ of men with seroconcordant partners said they knew their partner's status by getting tested together or talking with them; $5 \%$ determined partners' status indirectly and $14 \%$ did not report how they determined their partner's status. Seroconcordant UAI was more common among HIVpositive than HIV-negative men ( $53 \%$ vs $24 \%$, p <0.001). Among 288 HIV-negative men reporting UAI: seroconcordant UAI was more common with main vs casual partners $(76 \%$ vs $41 \%, \mathrm{p}<0.001)$ and within monogamous vs non-monogamous relationships (69\% vs $51 \%, p=0.003)$; serodiscordant UAI was more common among men with $\geq 3$ recent sex partners vs $<3$ partners ( $50 \%$ vs $33 \%, p=0.005$ ) and those who reported any anonymous sex partners vs none $(53 \%$ vs $28 \%, \mathrm{p}<0.001)$. Among men who said that they could not be talked out of safer sex with a seroconcordant partner, $10 \%$ of HIVnegative men and $16 \%$ of HIV-positive men reported serodiscordant UAI. $19 \%$ of HIV-negative men who endorsed seroconcordant partnerships reported serodiscordant UAI.

Conclusions Discrepancies between serosorting endorsement and practice underscore the importance of assessing cognitions related to risk-taking and behaviour. High rates of partner concurrency, infrequent testing, and indirect assessment of partner serostatus may limit the effectiveness of serosorting as a risk reduction strategy, even with main partners. The contribution of serosorting to HIV transmission within primary relationships warrants further research.

\section{2-S4.06 EXPLORING ENEMA PRACTICES AMONG MEN WHO HAVE SEX WITH MEN IN THE USA: IMPLICATIONS FOR SEXUAL HEALTH}

doi:10.1136/sextrans-2011-050109.90

\begin{abstract}
${ }^{1} \mathrm{~J}$ G. Rosenberger, ${ }^{1} \mathrm{D}$ Herbenick, ${ }^{1} \mathrm{~B}$ Van Der Pol, ${ }^{2} \mathrm{D} S$ Novak, ${ }^{1} \mathrm{M}$ Reece, ${ }^{3} \mathrm{~J} D$ Fortenberry. ${ }^{1}$ Indiana University, Bloomington, USA; ${ }^{2}$ Online Buddies Inc., Cambridge, USA; ${ }^{3}$ Indiana University School of Medicine, Indianapolis, USA
\end{abstract}

Purpose Recent literature has suggested that use of hygiene products (ie, douching) by women and men prior to sexual activity, changes the biological makeup of vaginal and anal tissue, potentially affecting risk for HIV/STI acquisition. While men who have sex with men (MSM) continue to be disproportionately impacted by HIV/STI, little information exists about men's lifetime enema behaviours, the situational contexts impacting their use, and attitudes towards use of such products.

Methods Data were collected via an internet survey from 27690 18-80 year old MSM. Measures included sociodemographics, recent/ lifetime sexual behaviour history, and items related to enema use.

Results Participants' median age was 39.0 years; ethnicities included white (84.5\%), Latino (6.4\%), African American (3.5\%); and, most $(79.9 \%)$ identified as homosexual. Over half $(54.2 \%)$ of men indicated that they were not currently in a romantic relationship and the majority $(88.9 \%)$ reported having two or more sexual partners in the past year. Most men (64.5\%) reported lifetime enema use, with
$33.8 \%$ reporting use in the past month. Most common reasons for starting enema use were to avoid feces during sex (72.5\%) and to feel clean during sex (65.6\%). Among enema users, $34.4 \%$ reported use of a disposable store bought enema, while $62.5 \%$ indicated having used a refillable enema or one that attached to the shower. Men somewhat or strongly agreed that enema use increased comfort with their anus $(75.8 \%)$, made sex more enjoyable $(75.5 \%)$, and increased the likelihood of being the receptive partner (75.8\%). During the most recent sexual event, enema use was reported both before (37.1\%) and after (8.1\%) sex, with most (42.0\%) reporting use $30 \mathrm{~min}-1 \mathrm{~h}$ prior to sex. Enema use at last sex was more likely to occur among men who were Caucasian $(p<0.01)$, who engaged in anal intercourse $(p<0.01)$, and whose sexual partner was someone they just met $(p<0.05)$.

Conclusions These data provide one of the first examinations of enema practices among MSM in the US. Findings highlight use of enemas prior to sexual activity as a common behaviour, and one associated with positively enhancing the sexual experience. The acceptability of enema use in this population may provide a vehicle for microbicide or virocide delivery once such compounds are fully developed. Future research should examine the relationship between behavioural practices and biological impacts of enema use in relation to HIV/STI.

\section{Social and behavioural aspects of prevention} oral session 5-Individual, Structural and Contextual Coping Strategies: Diverse causes and solutions

\section{2-S5.01 BONDING, BRIDGING, LINKING: EXPLORING RELATIONSHIP BETWEEN SOCIAL CAPITAL AND MIGRANTS' HIV RISK BEHAVIOUR AT DESTINATION}

doi:10.1136/sextrans-2011-050109.91

${ }^{1} \mathrm{D}$ Singh, ${ }^{2} \mathrm{~J}$ Blanchard, ${ }^{3} \mathrm{~J} \mathrm{O}^{\prime} \mathrm{Neil},{ }^{2} \mathrm{~J}$ Mignone, ${ }^{2} \mathrm{~S}$ Moses. ${ }^{1} \mathrm{PHFI}-$ Public Health Foundation of India, New Delhi, India; ${ }^{2}$ University of Manitoba, Winnipeg, Canada; ${ }^{3}$ Simon Frazer University, Canada

Background A multidimensional construct of social capital was employed to understand the relationship between social capital and migrants' HIV risk at destination place.

Methods The study was undertaken among Rajasthani migrants of age 18 and above in Mumbai and Ahmedabad in India to collect data from 1598 migrants through survey method and from 93 migrants through qualitative methods from January to June 2007. Social capital was measured in terms of three domains: bonding, bridging and linking. HIV risk had three measures: having casual partner in last 12 months in the city; sex with a sex worker in the city in last 12 months; and no or inconsistent condom use with a sex worker in last 12 months.

Results Migrants had substantial risk behaviour at destination: 385 respondents $(24.1 \%)$ reported having one or more casual partners; 218 migrants $(13.6 \%)$ had had sex with a sex worker; and 123 respondents $(7.7 \%)$ reported no or inconsistent condom use with a sex worker in the last 12 months in the city. Migrants reported higher risk in Ahmedabad compared to Mumbai for all the three risk measures: 251 (31.6\%) vs 134 (16.7\%) reported casual partners; 138 (17.4\%) vs 80 $(10 \%)$ reported having had sex with a sex worker; and $96(12.1 \%)$ vs $27(3.4 \%)$. Bonding and linking domains of social capital had higher values for migrants in Ahmedabad than Mumbai. Bridging social capital was higher in migrants in Mumbai as compared to migrants in Ahmedabad. All the components of bridging social capital had higher values for migrants in Mumbai than Ahmedabad. Bonding and linking social capital behaved differently in Mumbai and Ahmedabad. In Mumbai, migrants had lower HIV risk if they had high linking social capital and had higher risk if they had high bonding social 